\title{
Impact of neoadjuvant chemoradiotherapy on post-operative clinically significant pancreatic fistula: A systemic review and updated meta-analysis
}

\author{
Bhavin VASAVADA*, Hardik PATEL
}

Hepatobiliary and liver transplant, Consultant hepatobiliary and liver transplant, India

Introduction: The primary aim of our analysis was to do a systemic review and updated meta-analysis of literature published in the last 10 years and look for the association of neoadjuvant chemoradiation and risk of subsequent clinically significant pancreatic fistula. Methods: EMBASE, MEDLINE, and the Cochrane Database were searched for. Studies comparing outcomes in patients receiving neoadjuvant chemoradiotherapy first with those patients who received surgery first in case of pancreatic cancer. A systemic review and Metanalysis were done according to MOOSE and PRISMA guidelines. Heterogeneity was measured using Q tests and I2, and p < 0.10 was determined as significant.

Results: Twenty-six studies including 17,021 patients finally included in the analysis. 339 patients out of a total of 3,386 developed clinically significant pancreatic fistula in the neoadjuvant first group. 2,342 patients out of 13,335 patients developed clinically significant pancreatic fistula in the surgery first group. Neoadjuvant treatment significantly reduced the risk of subsequent clinically significant pancreatic fistula $(p \leq 0.0001)$. The number of patients with soft pancreas was significantly higher in the surgery first group $(p<$ $0.0001)$. Pancreatic duct diameter mentioned in only two studies but there was no significant difference between both groups $(p=1)$. Blood loss was significantly more in the surgery first group $(p<0.0001)$. There was no difference in pancreaticoduodenectomy or distal pancreatectomy performed between both groups $(p=0.82)$.

Conclusions: Neoadjuvant treatments may be responsible for the lower rates of clinically significant pancreatic fistula after subsequent surgery. 